\title{
Mecanismos envolvidos na trombogênese em pacientes com COVID-19 \\ Parte 1 - Trombogênese em SARS-CoV2: mecanismo da doença
}

\author{
Rodrigo Luppino Assad ${ }^{1}$
}

DOI: https://doi.org/10.46833/reumatologiasp.2020.19.3.19-21

\begin{abstract}
Resumo A infecção por coronavírus causadora da COVID-19 afetou milhões de pessoas em todo o mundo, com impacto no cotidiano da sociedade como um todo. Enfrentar com sucesso o desafio clínico da COVID-19 exige e continuará exigindo novos conhecimentos sobre a patogênese da doença e novos tratamentos para pacientes. Múltiplos acometimentos e características clínicas têm sido descritas relacionadas com a doença, e dentre estas a trombogênese está entre as mais prevalentes e associada a quadros mais graves, incluindo óbito. Achados necroscópicos de fenômenos trombóticos e embólicos têm sido descritos com frequência relativamente alta nestes pacientes. Os mecanismos envolvidos neste processo podem ser resumidos em ativação da enzima conversora de angiotensina 2 (ECA2) e lesão de células endoteliais, "tempestade de citocinas", imunotrombose (pelos linfócitos ou neutrófilos) ou até mesmo pela eventual formação de anticorpos antifosfolipídeos (SAF). Portanto, conforme conhecemos mais a fisiopatologia da COVID-19, maiores são as evidências de fenômenos trombóticos, e o conhecimento destes mecanismos nos remete a melhores opções de abordagem e maior sucesso terapêutico.
\end{abstract}

Unitermos COVID-19. Trombose. SAF. NETs.

\section{INTRODUÇÃO}

A infecção por coronavírus causadora da COVID-19 afetou milhões de pessoas em todo o mundo, com impacto no cotidiano da sociedade como um todo ${ }^{1}$.

Enfrentar com sucesso o desafio clínico da COVID-19 exige e continuará exigindo novos conhecimentos sobre a patogênese da doença e novos tratamentos para pacientes².

Múltiplos acometimentos e características clínicas têm sido descritas relacionadas com a doença, e dentre estas a trombogênese (Figura 1) está entre as mais prevalentes e associada a quadros mais graves, incluindo óbito ${ }^{3,4}$. Achados necroscópicos de fenômenos trombóticos e embólicos têm sido descritos com frequência relativamente alta nestes pacientes ${ }^{5,6}$.
Em recente estudo, realizado em 13 pacientes graves acometidos com o vírus, foi documentada a trombose in vivo, demostrando participação da trombose de pequenos vasos ${ }^{7}$.

\section{ATIVAÇÃO DA ENZIMA CONVERSORA DE ANGIOTENSINA 2 (ECA2) E LESÃO DE CÉLULAS ENDOTELIAIS}

A infecção pelo vírus SARS-CoV2 de células dos hospedeiros é a hipótese mais aceita a entrada do vírus pelos receptores da enzima conversora de angiotensina 2 (ECA2) ${ }^{8}$. A ativação anormal dos receptores pode levar ao estresse oxidativo, à agregação plaquetária e à trombose ${ }^{9}$.
1. Médico reumatologista pela Sociedade Brasileira de Reumatologia. Assistente da Disciplina de Reumatologia do Hospital das Clínicas da Faculdade de Medicina de Ribeirão Preto da Universidade de São Paulo (HC-FMRP-USP), Ribeirão Preto-SP, Brasil.

Correspondência: rodluppinoassad@gmail.com.

Como citar este artigo: Assad RL. Mecanismos envolvidos na trombogênese em pacientes com COVID-19. Parte 1 - Trombogênese em SARS-CoV2: mecanismo da doença. Rev Paul Reumatol. 2020 jul-set;19(3):19-21. DOI: https://doi.org/10.46833/ reumatologiasp.2020.19.3.19-21.

0 autor não contou com apoio financeiro.

0 autor declara não ter interesses associativos, comerciais, de propriedade ou financeiros que representem conflito de interesse nos produtos e empresas descritos neste artigo. 
Esses receptores estão presentes de forma abundante em células pulmonares, cardíacas, renais, intestinais e endoteliais ${ }^{10}$. 0 acometimento de células endoteliais, com lesão e exposição de fosfolipídeos da membrana, pode ativar o sistema de coagulação, levando à agregação plaquetária e à formação de trombos ${ }^{11}$.

$\mathrm{O}$ aumento de produtos de degradação de fibrina, como o dímero D, pode ser reflexo desta ativação do sistema de coagulação. Níveis aumentados nos pacientes estão relacionados com maior gravidade ${ }^{12}$.

Além disso, a lesão endotelial pode ser o gatilho para a formação de anticorpos contra esses fosfolipídeos, potencialmente os aPLs, questão discutida mais adiante, na Parte 2 deste estudo.

\section{"TEMPESTADE DE CITOCINAS"}

O processo inflamatório desencadeado pela infecção é a resposta imune para controle do processo infeccioso. Contudo, se este processo inflamatório não é controlado, ocorre processo contínuo inflamatório, com elevação de citocinas, em especial IL1b, IL6 e IL8.

Estas citocinas estão presentes em doenças inflamatórias agudas e crônicas e podem, por meio de estresse oxidativo e produção de espécies de reativas de oxigênio (ROS), causar hipercoagulabilidade por ativação plaquetária e aumento de trombopoitina ${ }^{13}$.

A IL-8 pode ser produzida por macrófagos, células epiteliais, do músculo liso das vias aéreas e células endoteliais. Existem muitos receptores na superfície da membrana capazes de se ligar à IL-8, inclusive em plaquetas, podendo ser esta uma das vias de ativação $0^{14}$.

Já a IL6 é uma citocina pleomórfica, e também tem potencial para ativar fibrinogênio e trombopoitina ${ }^{15}$.

\section{IMUNOTROMBOSE}

A ativação da coagulação pode ocorrer também de forma direta pela ativação de leucócitos ${ }^{16}$, ou pela formação de armadilhas extracelulares de neutrófilos (em inglês neutrophil extracelular trap - NET) (Figura 2).
Veras et al. demostraram que o vírus SARS-CoV-2 viável foi capaz de induzir neutrófilos a produzirem NETs, com antígenos virais sendo detectados dentro dos neutrófilos ${ }^{17}$.

Os neutrófilos ativados e, em particular, a formação de NET, contribuem para a propagação de trombos que afetam os leitos arteriais, venosos e vasculares microscópicos. Os eventos trombóticos são frequentes nos pacientes com COVID-19 e podem ser explicados somente pela formação de NETs². Mas as NETs têm sido também associadas e implicadas à SAF, portanto, além dos possíveis eventos trombóticos diretos causados por estas armadilhas, a ativação de anticorpos tem sido aventada ${ }^{18}$.

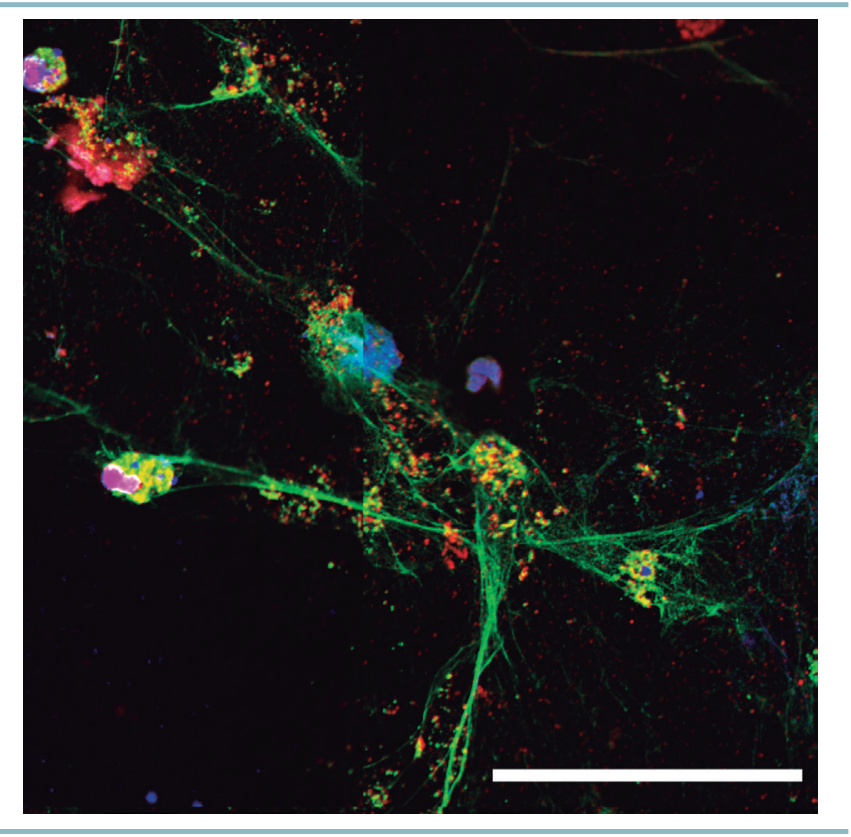

FIGURA 2 Exemplo de formação de NETs - Análise confocal representativa da liberação de NETs por neutrófilos isolados cultivados por $4 \mathrm{~h}$ a 37으. As células foram coradas para núcleos (DAPI, azul), mieloperoxidase (MPO, verde) e histona H3 citrulinada (H3Cit,

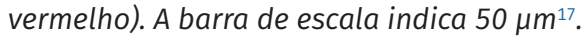
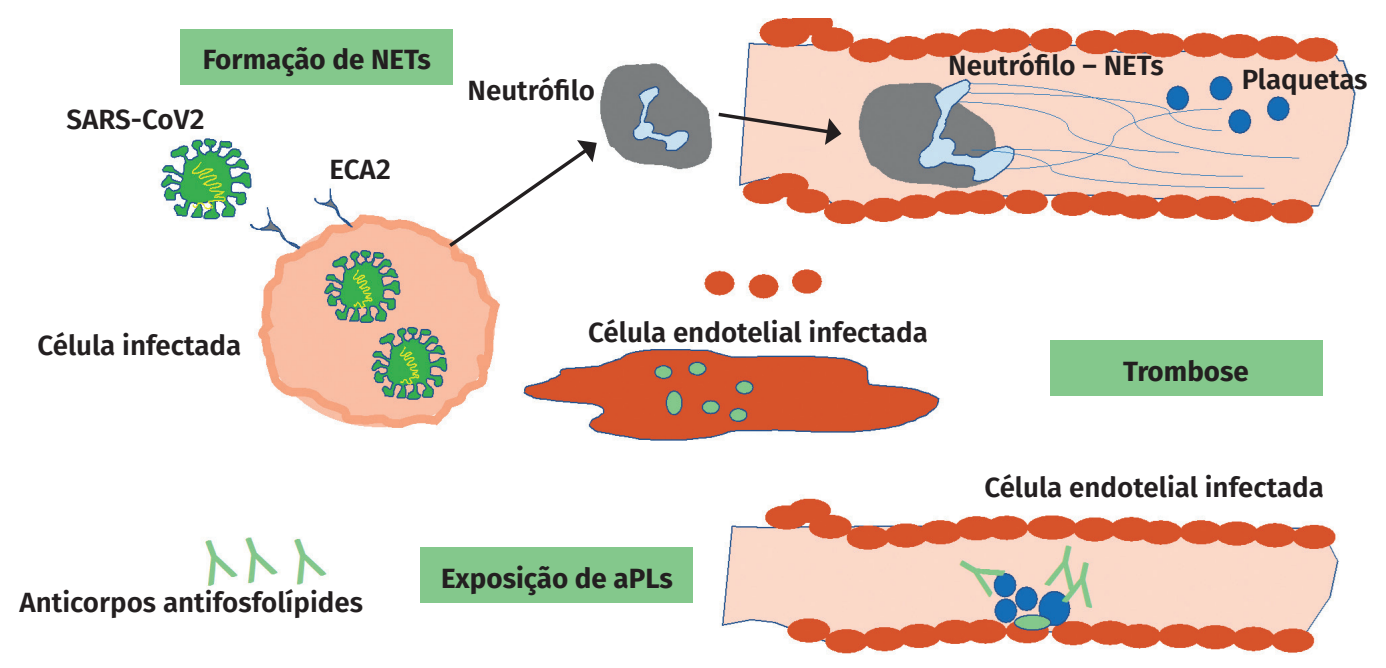

FIGURA 1 Esquema de dois possíveis mecanismos de trombose relacionada com a COVID-19. 


\section{NETS E SAF}

Outro possível mecanismo de associação entre as doenças talvez esteja relacionado com a fisiopatologia da SAF, em especial no processo inflamatório da SAF na gestação. Em modelos animais, a administração em camundongos grávidas de anticorpos policlonais IgG de indivíduos que têm SAF com altos títulos ou anticorpos antifosfolipídeos humanos monoclonais resulta em reabsorção fetal e restrição de crescimento ${ }^{19}$.

Os anticorpos anti- $\beta 2$-glicoproteína-1 desencadeiam a produção de citocinas pró-inflamatórias e quimiocinas (como IL-1, IL-7 e IL-8) via TLR4 em modelos animais, além da ativação do complemento e o recrutamento e estimulação de neutrófilos ${ }^{20}$.

Os neutrófilos também podem ser ativados diretamente por anticorpos anti- $\beta 2$-glicoproteína 1 que reconhecem a $\beta 2$-glicoproteína ligada à sua superfície celular e estimulam a formação de armadilha extracelular de neutrófilos (NET) por meio de mecanismos dependentes de espécies reativas de oxigênio e TLR $4^{18}$.

Pacientes com SAF apresentam formação aumentada de NET, depuração de NET prejudicada e maior número de granulócitos circulantes de baixa densidade, que têm uma capacidade aumentada de produzir citocinas e interferons tipo $1^{21}$. Este processo de produção de citocinas e quimiocinas é similar ao descrito na COVID-19, com ativação do inflamassoma ${ }^{22}$, assim como a formação de $\mathrm{NETs}^{17}$, e eferocitose inadequada destas células, com estimulação contínua do processo inflamatório.

Tais achados têm implicação na prática clínica, com eventuais proposições para tratamento, e eventual bloqueio do processo, como no uso de colchicina ${ }^{23}$.

Apesar destas concordâncias entre os mecanismos fisiopatológicos entre as doenças, que embasam o raciocínio para melhor entendimento das doenças, como é sabido, achados concomitantes podem ser somente epifenômenos. Portanto, apesar dos recentes e grandes avanços no entendimento das duas doenças (SAF e COVID-19), é necessária a análise crítica das evidências, abordadas na Parte 2 deste estudo, apresentada a seguir.

Quanto à trombogênese em SARS-CoV2, melhores e mais confiáveis evidências têm sido apresentadas sobre o mecanismo da doença. Com o maior conhecimento da etiopatogenia, mais clara fica a associação com os fenômenos trombóticos. O conhecimento destes mecanismos nos remete a melhores opções de abordagens e maior sucesso terapêutico.

\section{Referências}

1. Dong $\mathrm{E}, \mathrm{Du} \mathrm{H}$, Gardner L. An interactive web-based dashboard to track COVID-19 in real time. Lancet Infect Dis. 2020;20(5):533-4. https://doi. org/10.1016/S1473-3099(20)30120-1.

2. Middleton EA, He X-Y, Denorme F, Campbell RA, Ng D, Salvatore SP, et al. Neutrophil extracellular traps contribute to immunothrombosis in COVID-19 acute respiratory distress syndrome Blood. 2020;136(10):1169-79. https:// doi.org/10.1182/blood.2020007008.

3. Tang N, Li D, Wang X, Sun Z. Abnormal coagulation parameters are associated with poor prognosis in patients with novel coronavirus pneumonia. J Thromb Haemost. 2020;18(4):844-7. https://doi.org/10.1111/jth.14768.

4. Cui S, Chen S, Li X, Liu S, Wang F. Prevalence of venous thromboembolism in patients with severe novel coronavirus pneumonia. J Thromb Haemost. 2020;18(6):1421-4. https://doi.org/10.1111/jth.14830.

5. Dolhnikoff M, Duarte-Neto AN, Monteiro RAA, Silva LFF, Oliveira EP, Saldiva PHN, et al. Pathological evidence of pulmonary thrombotic phenomena in severe COVID-19. J Thromb Haemost. 2020 Jun;18(6):1517-9. https://doi. org/10.1111/jth.14844.

6. Ackermann M, Verleden SE, Kuehnel M, Haverich A, Welte T, Laenger F, et al. Pulmonary Vascular Endothelialitis, Thrombosis, and Angiogenesis in Covid- 19. N Engl J Med. 2020 Jul 9;383(2):120-8. https://doi.org/10.1056/NEJMoa2015432.

7. Espírito-Santo DA, Lemos $\mathrm{ACB}$, Miranda $\mathrm{CH}$. In vivo demonstration of microvascular thrombosis in severe Covid-19. J Thromb Thrombolysis. 2020 Nov;50(4):790-4. https://doi.org/10.1007/s11239-020-02245-x.

8. Wrapp D, Wang N, Corbett KS, Goldsmith JA, Hsieh CL, Abiona O, et al. Cryo-EM structure of the 2019-nCoV spike in the prefusion conformation. Science. 2020 Mar 13;367(6483):1260-63. Epub 2020 Feb 19. https://doi. org/10.1126/science.abb2507.

9. Fraga-Silva RA, da Silva DG, Montecucco F, Mach F, Stergiopulos N, da Silva RF, et al. The angiotensin-converting enzyme 2/angiotensin-(1-7)/mas receptor axis: a potential target for treating thrombotic diseases. Thromb Haemost. 2012;108:1089-96. https://doi.org/10.1160/TH12-06-0396.

10. Bertram S, Heurich A, Lavender H, Gierer S, Danisch S, Perin P, et al. Influenza and SARS-Coronavirus Activating Proteases TMPRSS2 and HAT Are Expressed at Multiple Sites in Human Respiratory and Gastrointestinal Tracts. PLoS One. 2012;7(4):e35876. Epub 2012 Apr 30. https://doi.org/10.1371/journal. pone.0035876.

11. Spiezia L, Boscolo A, Poletto F, Cerruti L, Tiberio I, Campello E, et al. COVID-19-related severe hypercoagulability in patients admitted to intensive care unit for acute respiratory failure. Thromb Haemost. 2020 Jun;120(6):9981000. Epub 2020 Apr 21. https://doi.org/10.1055/s-0040-1710018.

12. Gao Y, Li T, Han M, Li X, Wu D, Xu Y, et al. Diagnostic utility of clinical laboratory data determinations for patients with the severe COVID-19. J Med Virol. 2020;92:791-6. https://doi.org/10.1002/jmv.25770.
13. Bester J, Pretorius E. Effects of IL-1 $1 \beta$, IL- 6 and IL- 8 on erythrocytes, platelets and clot viscoelasticity. Sci. Rep. 6, 32188. https://doi.org/10.1038/ srep32188.

14. Regnault V, de Maistre E, Carteaux J-P, Gruel Y, Nguyen P, Tardy B, et al Platelet activation induced by human antibodies to interleukin-8. Blood. 2003 Feb 15;101(4):1419-21. Epub 2002 Oct 10. https://doi.org/10.1182/ blood-2002-02-0620.

15. Tanaka T, Narazaki M, Kishimoto T. Immunotherapeutic implications of IL-6 blockade for cytokine storm. Immunotherapy. 2016;8:959-70. https://doi. org/10.2217/imt-2016-0020.

16. Barnes BJ, Adrover JM, Baxter-Stoltzfus A, Borczuk A, Cools-Lartigue J, Crawford JM, et al. Targeting potential drivers of COVID-19: Neutrophil extracellular traps. J Exp Med. 2020 Jun 1;217(6):e20200652. https://doi. org/10.1084/jem.20200652.

17. Veras FP, Pontelli M, Silva C, Toller-Kawahisa J, Lima M, Nascimento D, et al. SARS-CoV-2 triggered neutrophil extracellular traps (NETs) mediate COVID-19 pathology. J Exp Med. 2020 Dec 7;217(12):e20201129. https://doi. org/10.1084/jem.20201129.

18. Yalavarthi S, Gould TJ, Rao AN, Mazza LF, Morris AE, Núñez-Álvarez C, et al. Release of neutrophil extracellular traps by neutrophils stimulated with antiphospholipid antibodies: a newly identified mechanism of thrombosis in the antiphospholipid syndrome. Arthritis Rheumatol. 2015 Nov;67(11):29903003. https://doi.org/10.1002/art.39247.

19. Holers VM, Girardi G, Mo L, Guthridge JM, Molina H, Pierangeli SS, et al. Complement C3 activation is required for antiphospholipid antibody-induced fetal loss. J Exp Med. 2002 Jan 21;195(2):211-20. https://doi.org/10.1084/ jem.200116116.

20. Mulla MJ, Brosens JJ, Chamley LW, Giles I, Pericleous C, Rahman A, et al. Antiphospholipid antibodies induce a pro-inflammatory response in first trimester trophoblast via the TLR4/MyD88 pathway. Am J Reprod Immunol. 2009 Aug;62(2):96-111. https://doi.org/10.1111/j.16000897.2009.00717.x.

21. Marder W, Knight JS, Kaplan MJ, Somers EC, Zhang X, O'Dell AA, et al. Placental histology and neutrophil extracellular traps in lupus and pre-eclampsia pregnancies. Lupus Sci Med. 2016 Apr 27;3(1):eoo0134. eCollection 2016. https://doi.org/10.1136/lupus-2015-000134.

22. Rodrigues TS, Keyla SG Sa, Ishimoto AY, Becerra A, Oliveira S, Almeida L, et al. Inflammasome activation in COVID-19 patients. 2020 Aug 6. medRxiv 2020.08.05.20168872. https://doi.org/10.1101/2020.08.05.20168872.

23. Lopes MIF, Bonjorno LP, Giannini MC, Amaral NB, Benatti MN, Rezek UC, et al Beneficial effects of colchicine for moderate to severe COVID-19: an interim analysis of a randomized, double-blinded, placebo controlled clinical trial. 2020 Aug 12. medRxiv 2020.08.06.20169573. https://doi.org/10.1101/2020 .08.06.20169573. 